Pacific

Journal of

Mathematics

LIOUVILLE TYPE THEOREMS FOR THE $p$-HARMONIC FUNCTIONS ON CERTAIN MANIFOLDS

JING YI CHEN AND YUE WANG 


\title{
LIOUVILLE TYPE THEOREMS FOR THE $p$-HARMONIC FUNCTIONS ON CERTAIN MANIFOLDS
}

\author{
JiNGYI CHEN AND YUE WANG
}

\begin{abstract}
We show that for a certain range of $p>n$, the Dirichlet problem at infinity is unsolvable for the $p$-Laplace equation for any nonconstant continuous boundary data on an $\boldsymbol{n}$-dimensional Cartan-Hadamard manifold constructed from a complete noncompact shrinking gradient Ricci soliton. Using the steady gradient Ricci soliton, we find an incomplete Riemannian metric on $\mathbb{R}^{2}$ with positive Gauss curvature such that every positive $p$ harmonic function must be constant for $p \geq 4$.
\end{abstract}

\section{Introduction}

In this article, we study two questions about the $p$-Laplace equation on Riemannian manifolds. The first one is the solvability of the Dirichlet problem at infinity on a negatively curved complete noncompact manifold, and the second one is the Liouville property for positive solutions on $\mathbb{R}^{2}$ equipped with an incomplete metric with positive Gauss curvature. In both cases, the $n$-dimensional manifold $M$ under consideration is equipped with a Riemannian metric $e^{2 f /(p-n)} g$ where $(M, g, f)$ is a complete gradient Ricci soliton which is shrinking for the first case and steady for the second case.

On a Riemannian manifold, for a constant $p>1$, a function $v$ in $W_{\mathrm{loc}}^{1, p} \cap L_{\mathrm{loc}}^{\infty}$ is $p$-harmonic if it is a weak solution to the $p$-Laplacian equation

$$
\operatorname{div}\left(|\nabla v|^{p-2} \nabla v\right)=0 .
$$

It is known that $p$-harmonic functions are in $C^{1, \alpha}$ (see [Tolksdorf 1984] and the references therein).

Chen is partially supported by NSERC (RGPIN 203199-1). Wang is supported in part by the National Natural Science Foundation of China (No. 10901147) and Zhejiang Provincial Natural Science Foundation of China (No. LY12A01028), and she is grateful to PIMS/UBC for hosting her visit during which part of this work was carried out.

MSC2010: 53C21, 58J05.

Keywords: Liouville type theorems, $p$-harmonic functions, gradient Ricci solitons. 
The behavior of harmonic and, more generally, $p$-harmonic functions depends on the sign of the curvature of the manifold in an essential way. Therefore, we must treat negatively curved and nonnegatively curved manifolds separately.

A Cartan-Hadamard manifold is a complete simply connected Riemannian manifold with nonpositive sectional curvature everywhere. It is well-known that a Cartan-Hadamard manifold $M$ can be compactified by attaching a sphere $M(\infty)$ at infinity. In the cone topology, the compactification is homeomorphic to a closed Euclidean $n$-ball [Eberlein and O'Neill 1973]. The Dirichlet problem at infinity for $p$-harmonic functions is to solve the $p$-Laplace equation (1-1) on $M$ such that $v$ agrees with a given continuous function $\varphi$ on $M(\infty)$. For $p=2$, the Dirichlet problem at infinity for harmonic functions is solvable if there are suitable lower and upper bounds for the sectional curvature [Anderson 1983; Anderson and Schoen 1985; Choi 1984; Hsu 2003; Sullivan 1983]. Ancona [1994] constructed an example showing that the Dirichlet problem is unsolvable if only a negative constant upper bound is imposed. For $p \in(1, \infty)$, the Dirichlet problem at infinity is solvable under similar curvature assumptions like those in the case $p=2$; in particular, it is solvable if the sectional curvature is bounded by

$$
-r^{2 \alpha-4-\epsilon} \leq K \leq-\frac{\alpha(\alpha-1)}{r^{2}}
$$

near $M(\infty)$ where $\epsilon>0$ and $\alpha>1$, where $r$ is the distance to a fixed point, and for $p \in(1,1+(n-1) \alpha)$ [Holopainen 2002; Holopainen and Vähäkangas 2007; Pansu 1989].

Our first result is to show the unsolvability of the Dirichlet problem at infinity on certain Cartan-Hadamard manifolds constructed from shrinking gradient Ricci solitons, for a certain range of $p>n$. In particular, the unsolvability holds for the shrinking Gaussian soliton $\left(\mathbb{R}^{n}, d x^{2},|x|^{2} / 4\right)$ for every $p>n$. It is interesting to observe that the sectional curvature of the complete negatively curved metric $e^{|x|^{2} /(2(p-n))} d x^{2}$ is not bounded above by $-\alpha(\alpha-1) / r^{2}$, for any constant $\alpha>1$, at certain sections for sufficiently large $r$ (see remark on page 319). This indicates the upper bound in (1-2) is sharp in some sense for the solvability of the Dirichlet problem at infinity.

Theorem 1.1. Suppose that $(M, g, f)$ is a simply connected n-dimensional complete noncompact shrinking gradient Ricci soliton whose sectional curvatures are bounded above by a constant $K_{0}$ with $0<K_{0}<1 /(2(n-1))$. Then the Dirichlet problem at infinity for the $p$-Laplace equation on $\left(M, e^{2 f /(p-n)} g\right)$ is unsolvable for any nonconstant continuous boundary value $\varphi$ and $n<p<\frac{1}{K_{0}}+2-n$.

The proof relies on a Liouville type property (Proposition 2.1) for positive solutions to the $p$-Laplace equation on $\left(M, e^{-2 f /(n-p)} g\right)$ for every $p>1$, where Cao and Zhou's [2010] estimates on $f$ and on the volume growth for gradient 
shrinking Ricci solitons are crucial as they imply that $e^{-f}$ is integrable on $(M, g)$. The advantage for considering the range $p>n$ is that, under the conformal change of metric, it yields a complete metric $\tilde{g}$ and it guarantees the negativity of the curvature of $\tilde{g}$ under the curvature assumption $K \leq K_{0}$, while one does not have such flexibility for $p=2$.

However, the integration argument in the proof of Proposition 2.1 is no longer valid for steady gradient Ricci solitons due to different behavior of $f$ (typically $f$ tends to $-\infty$ along a sequence of points $x_{k}$ that go to infinity [Munteanu and Sesum 2013; Wu 2013]). Alternatively, a powerful way to prove Liouville type theorems for positive harmonic functions on complete manifolds with nonnegative Ricci curvature is via Yau's gradient estimate [1975]. The $p$-harmonic version of Yau's estimate is established by Wang and Zhang [2011] (see [Sung and Wang 2014] for a sharp form of the estimate). For a positive $p$-harmonic function $u$ in the conformally changed metric $\tilde{g}=e^{-2 f /(n-p)} g$, we first derive a maximum principle for $|\nabla \log u|$ for steady (or shrinking) gradient Ricci solitons, via a Bochner type formula. However, the required assumption on Ricci curvature for the gradient estimates cannot hold globally for steady gradient Ricci solitons if $\operatorname{dim} M>2$ because it would imply that the scalar curvature of $g$ possesses a positive constant lower bound. But this is impossible as shown in [Munteanu and Sesum 2013; Wu 2013]. In dimension 2, we can combine the maximum principle (Proposition 3.3) and the gradient estimate to prove a Liouville type result on the 2-plane with a positively curved incomplete metric.

Theorem 1.2. Let $\left(\mathbb{R}^{2}, g, f\right)$ be Hamilton's cigar soliton. Then there does not exist any nonconstant positive $p$-harmonic function on $\left(\mathbb{R}^{2}, \tilde{g}\right)$ for $p \geq 4$.

Harmonic functions on the complete gradient Ricci solitons have been studied by Munteanu and Sesum [2013] and Munteanu and Wang [2012] with applications to the geometry and topology of the solitons. Moser [2007] observed an interesting connection between the inverse mean curvature flow formulated as level sets in $\mathbb{R}^{n}$ and 1-harmonic functions. Kotschwar and Ni [2009] generalize this to Riemannian ambient manifolds. There is also recent work on gradient estimates for weighted $p$-harmonic functions and the first $p$-eigenfunctions [Dung and Dat 2015].

\section{The Dirichlet problem at infinity}

In this section, the triple $(M, g, f)$ is assumed to be a complete noncompact shrinking gradient Ricci soliton. We first establish the following Liouville property for positive $p$-harmonic functions for $p>1$ with no additional curvature assumption.

An $n$-dimensional Riemannian manifold $(M, g)$ is a gradient Ricci soliton if

$\operatorname{Ric}+\nabla \nabla f+\varepsilon g=0$ 
for some smooth function $f$ and $\varepsilon=-\frac{1}{2}, 0, \frac{1}{2}$. Corresponding to the three values of $\varepsilon$, the gradient Ricci soliton $(M, g, f)$ is shrinking, steady, or expanding [Chow et al. 2006; Hamilton 1995].

Proposition 2.1. Let $(M, g, f)$ be a complete noncompact gradient shrinking Ricci soliton. Then there is no nonconstant positive p-harmonic function on $\left(M, e^{-2 f /(n-p)} g\right)$ for $p>1$.

Proof. Since $u$ is a $p$-harmonic function on $(M, \tilde{g})$ where $\tilde{g}=e^{-2 f /(n-p)} g$,

$$
\operatorname{div}_{\tilde{g}}\left(|\widetilde{\nabla} w|_{\tilde{g}}^{p-2} \widetilde{\nabla} w\right)=|\widetilde{\nabla} w|_{\tilde{g}}^{p}
$$

holds for $w=-(p-1) \log u$. For any smooth cut-off function $\phi \in C_{0}^{\infty}(M)$, in the complete metric $g$, we require

$$
\begin{cases}\phi=1 & \text { on } B_{x_{0}}(\rho, g), \\ \phi=0 & \text { on } M \backslash B_{x_{0}}(2 \rho, g), \\ 0 \leq \phi \leq 1 & \text { on } M, \\ |\nabla \phi|^{2} \leq C / \rho^{2} & \text { on } M .\end{cases}
$$

Here $B_{x_{0}}(r, g)$ stands for the geodesic ball centered at $x_{0}$ with radius $r$ in the metric $g$ in $M$. Multiplying (2-2) by $\phi^{2}$, then integrating and applying Stokes' theorem, we have

$$
\begin{aligned}
\int_{M}|\widetilde{\nabla} w|_{\tilde{g}}^{p} \phi^{2} d \mu_{\tilde{g}} & =-2 \int_{M} \phi|\widetilde{\nabla} w|_{\tilde{g}}^{p-2} \widetilde{\nabla} w \widetilde{\nabla} \phi d \mu_{\tilde{g}} \\
& \leq 2\left(\int_{M} \phi^{2}|\widetilde{\nabla} w|_{\tilde{g}}^{p} d \mu_{\tilde{g}}\right)^{(p-1) / p}\left(\int_{M} \phi^{2}|\widetilde{\nabla} \phi|_{\tilde{g}}^{p} d \mu_{\tilde{g}}\right)^{1 / p}
\end{aligned}
$$

by the Cauchy-Schwarz inequality $(p>1)$. Therefore, we have

$$
\int_{M} \phi^{2}|\widetilde{\nabla} w|_{\tilde{g}}^{p} d \mu_{\tilde{g}} \leq 2^{p} \int_{M} \phi^{2}|\widetilde{\nabla} \phi|_{\tilde{g}}^{p} d \mu_{\tilde{g}}
$$

Converting back to the metric $g$, we are led to

$$
\int_{M} \phi^{2}|\nabla w|^{p} e^{-f} d \mu_{g} \leq 2^{p} \int_{M} \phi^{2}|\nabla \phi|^{p} e^{-f} d \mu_{g}
$$

By Theorem 1.1 in [Cao and Zhou 2010], the potential function $f$ for a shrinking gradient Ricci soliton satisfies the pointwise estimate

$$
\frac{1}{4}(r(x)-c)^{2} \leq f(x) \leq \frac{1}{4}(r(x)+c)^{2}
$$

for $x \in M \backslash B_{x_{0}}(1, g)$, where $r(x)$ is the distance from $x$ to a fixed point $x_{0}$ in $M$ and $c$ is a positive constant. 
Therefore, by (2-3) and (2-4),

$$
\begin{aligned}
\int_{B\left(x_{0}, \rho\right)}|\nabla w|^{p} e^{-(r+c)^{2} / 4} d \mu_{g} & \leq \int_{M} \phi^{2}|\nabla w|^{p} e^{-f} d \mu_{g} \\
& \leq \frac{2^{p} C e^{-(\rho-c)^{2} / 4}}{\rho^{p}} \int_{B_{x_{0}}(2 \rho, g) \backslash B_{x_{0}}(\rho, g)} d \mu_{g} \\
& \leq \frac{2^{p} C e^{-(\rho-c)^{2} / 4}}{\rho^{p}} \rho^{n}
\end{aligned}
$$

where the last inequality follows from the volume growth estimate (Theorem 1.2 in [Cao and Zhou 2010]) on shrinking gradient Ricci solitons:

$$
\operatorname{Vol}\left(B_{x_{0}}(\rho, g)\right) \leq C \rho^{n}
$$

for sufficiently large $\rho$ and uniform constant $C$. Now letting $\rho \rightarrow \infty$, we conclude $|\nabla w| \equiv 0$ on $M$, so $u$ is a constant.

Next, we show that $(M, \tilde{g})$ can be turned into a negatively curved manifold under suitable assumptions on $p$ and the sectional curvature $K$ of $(M, g)$.

Proposition 2.2. Let $(M, g, f)$ be a simply connected $n$-dimensional complete noncompact shrinking gradient Ricci soliton whose sectional curvature is bounded above by a constant $K_{0}$ with $0<K_{0}<1 /(2(n-1))$. Then $\left(M, e^{-2 f /(n-p)} g\right)$ is a Cartan-Hadamard manifold for $n<p \leq \frac{1}{K_{0}}+2-n$.

Proof. When $p>n$, the metric $\tilde{g}=e^{-2 f /(n-p)} g$ is complete since

$$
-\frac{2 f(x)}{n-p}=\frac{2 f(x)}{p-n} \geq \frac{(r-c)^{2}}{2(p-n)}
$$

by [Cao and Zhou 2010] and completeness of $g$.

We use the conventions in [Chow et al. 2006] for curvatures. The Riemann curvature tensor is written as

$$
\begin{aligned}
R\left(\frac{\partial}{\partial x^{i}}, \frac{\partial}{\partial x^{j}}\right) \frac{\partial}{\partial x^{k}} & =R_{i j k}^{l} \frac{\partial}{\partial x^{l}} \\
R_{i j k l} & =\left\langle R\left(\frac{\partial}{\partial x^{i}}, \frac{\partial}{\partial x^{j}}\right) \frac{\partial}{\partial x^{k}}, \frac{\partial}{\partial x^{l}}\right\rangle
\end{aligned}
$$

and if $\partial / \partial x^{1}, \ldots, \partial / \partial x^{n}$ is orthonormal at $x_{0} \in M$, then the sectional curvature of the plane $P_{i j}$ spanned by $\partial / \partial x^{i}, \partial / \partial x^{j}$ at $x_{0}$ is

$$
K\left(P_{i j}\right)=R_{i j j i}
$$

and the Ricci curvature at $x_{0}$ is

$$
R_{j k}=\sum_{i=1}^{n} R_{i j k}^{i} .
$$


Under the conformal change of metric $\tilde{g}=e^{2 f /(p-n)} g$, the sectional curvature at $x_{0}$ becomes

$$
\begin{aligned}
\widetilde{K}\left(P_{i j}\right) & =\frac{\tilde{g}\left(\widetilde{R}_{i j j}^{s} \frac{\partial}{\partial x^{s}}, \frac{\partial}{\partial x^{i}}\right)}{\tilde{g}_{i i} \tilde{g}_{j j}-\tilde{g}_{i j}^{2}} \\
& =e^{4 f /(n-p)} \widetilde{R}_{i j j i} \\
& =e^{4 f /(n-p)} \cdot e^{2 f /(p-n)}\left(R_{i j j i}-\frac{f_{i i}+f_{j j}}{p-n}-\frac{|\nabla f|^{2}-f_{i}^{2}-f_{j}^{2}}{(p-n)^{2}}\right) \\
& =e^{2 f /(n-p)}\left(K\left(P_{i j}\right)-\frac{f_{i i}+f_{j j}}{p-n}-\frac{|\nabla f|^{2}-f_{i}^{2}-f_{j}^{2}}{(p-n)^{2}}\right)
\end{aligned}
$$

(see p. 27 in [Chow et al. 2006]). On the gradient shrinking Ricci soliton, we therefore have

$$
\widetilde{K}\left(P_{i j}\right) \leq e^{2 f /(n-p)}\left(K\left(P_{i j}\right)+\frac{R_{i i}+R_{j j}-1}{p-n}\right)
$$

by using the defining equation for shrinking gradient Ricci solitons and dropping the last term above that is nonpositive for $i \neq j$.

From the assumption on $K_{0}$ and $p>n$, it follows that

$$
\begin{aligned}
K\left(P_{i j}\right)+\frac{R_{i i}+R_{j j}-1}{p-n} & =K\left(P_{i j}\right)+\frac{\sum_{s \neq i} K\left(P_{i s}\right)+\sum_{s \neq j} K\left(P_{s j}\right)-1}{p-n} \\
& \leq\left(1+\frac{2(n-1)}{p-n}\right) K_{0}-\frac{1}{p-n} \\
& \leq \frac{1}{p-n}\left((p+n-2) K_{0}-1\right) .
\end{aligned}
$$

Therefore, we conclude that the sectional curvature $\widetilde{K}$ of $\left(M, e^{2 f /(p-n)} g\right)$ is nonpositive since $p+n-2 \leq \frac{1}{K_{0}}$.

Proof of Theorem 1.1. Suppose there is a solution $u$ to the Dirichlet problem at infinity and $u=\varphi$ on $M(\infty)$ for some nonconstant function $\varphi \in C^{0}(M(\infty))$. Then $u$ is continuous on $M \cup M(\infty)$, hence it is bounded. Then $u-\inf _{M} u+1$ is a positive solution to the $p$-Laplace equation on $(M, \tilde{g})$, therefore it must be constant from Proposition 2.1. Thus, $u$ is constant on $M$ and $\varphi$ must be constant on $M(\infty)$. The contradiction concludes the proof.

When $\mathbb{R}^{n}$ is viewed as a shrinking gradient Ricci soliton with $f(x)=|x|^{2} / 4$, we can take $K_{0}=0$ and obtain the following corollary.

Corollary 2.3. The Dirichlet problem at infinity for the p-Laplace equation is unsolvable on $\left(\mathbb{R}^{n}, e^{|x|^{2} /(2(p-n))} d x^{2}\right)$ for every $p>n$. 
Remark. The sectional curvature of $\tilde{g}=e^{2|x|^{2} /(4(p-n))} d x^{2}$ can be computed from (2-5):

$$
\widetilde{K}\left(P_{i j}\right)(x)=-e^{-|x|^{2} /(2(p-n))}\left(\frac{1}{p-n}+\frac{|x|^{2}-\left(x^{i}\right)^{2}-\left(x^{j}\right)^{2}}{4(p-n)^{2}}\right)
$$

where $P_{i j}(x)$ is the plane spanned by $\left\{\partial / \partial x^{i}, \partial / \partial x^{j}\right\}$ at $x \in \mathbb{R}^{n}$. The Riemannian distance from $x$ to the origin is

$$
r(x)=\int_{0}^{|x|} e^{s^{2} /(4(p-n))} d s .
$$

If we take $x=\left(0, \ldots, 0, x^{i}, 0, \ldots, 0\right)$, then $|x|^{2}-\left(x^{i}\right)^{2}-\left(x^{j}\right)^{2}=0$ and

$$
\begin{aligned}
\lim _{|x| \rightarrow \infty}-\widetilde{K}\left(P_{i j}(x)\right) r^{2}(x) & =\lim _{|x| \rightarrow \infty} \frac{\left(\int_{0}^{|x|} e^{s^{2} /(4(p-n))} d s\right)^{2}}{(p-n) e^{|x|^{2} /(2(p-n))}} \\
& =\frac{1}{p-n}\left(\lim _{|x| \rightarrow \infty} \frac{2(p-n)}{|x|}\right)^{2}=0
\end{aligned}
$$

by l'Hôpital's rule. This in particular shows that there does not exist a constant $\alpha>1$ for which

$$
K(x) \leq-\frac{\alpha(\alpha-1)}{r^{2}(x)}
$$

for all sections at $x$ for large $r(x)$.

\section{A Liouville theorem on $\mathbb{R}^{2}$ with an incomplete metric with positive curvature}

In this section, we consider the $p$-Laplace equation weighted by a smooth function $f$ on a manifold $(M, g)$, which is equivalent to the $p$-Laplace equation on $\left(M, e^{-2 f /(n-p)} g\right)$, and derive a Bochner formula for its solutions. Specialized to the shrinking or steady gradient Ricci solitons, the Bochner formula yields a maximum principle, and this is applied to Hamilton's cigar soliton.

A Bochner type formula for the weighted p-Laplace equation. Let $g$ be a Riemannian metric on an $n$-dimensional manifold $M$, and let $f$ be a smooth realvalued function on $M$. Consider the equation

$$
\operatorname{div}\left(|\nabla u|^{p-2} \nabla u\right)-|\nabla u|^{p-2}\langle\nabla f, \nabla u\rangle=0
$$

on $M$. This equation has a variational structure; in fact, it is the Euler-Lagrange equation of the weighted $p$-energy functional

$$
E_{p, f}(u)=\int_{M}|\nabla u|^{p} e^{-f} d \mu_{g}
$$

We call (3-1) the $f$-weighted $p$-Laplacian equation on $(M, g)$. 
Proposition 3.1. Under a conformal change $\tilde{g}=e^{-2 f /(n-p)} g$, $u$ is a solution to (3-1) on $(M, g)$ if and only if $u$ is a solution to the $p$-Laplace equation (1-1) on $(M, \tilde{g})$.

Proof. We write $\nabla$ for $\nabla_{g}$ and $\widetilde{\nabla}$ for $\nabla_{\tilde{g}}$. For any $\varphi \in C_{0}^{\infty}(M)$,

$$
\begin{aligned}
\int_{M}\left\langle\widetilde{\nabla} \varphi,|\widetilde{\nabla} u|_{\tilde{g}}^{p-2}\right. & \widetilde{\nabla} u\rangle_{\tilde{g}} d \mu_{\tilde{g}} \\
& =\int_{M}|\widetilde{\nabla} u|_{\tilde{g}}^{p-2}\langle\widetilde{\nabla} \varphi, \widetilde{\nabla} u\rangle_{\tilde{g}} d \mu_{\tilde{g}} \\
& =\int_{M}\left(e^{(p-2) f /(n-p)}|\nabla u|_{g}^{p-2}\right) e^{2 f /(n-p)}\langle\nabla \varphi, \nabla u\rangle_{g} e^{-n f /(n-p)} d \mu_{g} \\
& =\int_{M}\left\langle\nabla \varphi,|\nabla u|_{g}^{p-2} \nabla u\right\rangle_{g} e^{-f} d \mu_{g} .
\end{aligned}
$$

This shows that any weak solution to $(3-1)$ on $(M, g)$ is also a weak solution to $(1-1)$ on $(M, \tilde{g})$ and vice versa.

Suppose $u(x, t)$ is a positive solution of (3-1). Define

$$
\begin{aligned}
& w=-(p-1) \log u, \\
& h=|\nabla w|^{2} .
\end{aligned}
$$

We consider the symmetric $n \times n$ matrix

$$
A=\mathrm{id}+(p-2) \frac{\nabla w \otimes \nabla w}{h} .
$$

Note that $A$ is well defined whenever $h>0$ and is positive definite for $p>1$. Arising from the linearized operator of the nonlinear $p$-harmonic equations, this matrix was first introduced in [Moser 2007] and was used in [Kotschwar and Ni 2009; Wang and Zhang 2011] to study positive $p$-harmonic functions.

For the $f$-weighted $p$-Laplace equation (3-1), the linearized operator is

$$
\mathcal{L}(\psi)=\operatorname{div}\left(h^{\frac{p}{2}-1} A(\nabla \psi)\right)-h^{\frac{p}{2}-1}\langle\nabla f, A(\nabla \psi)\rangle-p h^{\frac{p}{2}-1}\langle\nabla w, \nabla \psi\rangle
$$

for smooth functions $\psi$ on $M$, and the following Bochner type formula holds.

Proposition 3.2. Let $u$ be a positive smooth solution to (3-1) in an open subset $U$ in $M$ and assume $h>0$ on $U$. Then

(3-2) $\operatorname{div}\left(h^{\frac{p}{2}-1} A(\nabla h)\right)-h^{\frac{p}{2}-1}\langle\nabla f, A(\nabla h)\rangle-p h^{\frac{p}{2}-1}\langle\nabla w, \nabla h\rangle$

$$
=\left(\frac{p}{2}-1\right)|\nabla h|^{2} h^{\frac{p}{2}-2}+2 h^{\frac{p}{2}-1}\left(|\nabla \nabla w|^{2}+\operatorname{Ric}(\nabla w, \nabla w)+\nabla \nabla f(\nabla w, \nabla w)\right) .
$$


Proof. Using (3-1), we first observe

(3-3) $\operatorname{div}\left(|\nabla w|^{p-2} \nabla w\right)-|\nabla w|^{p}$

$$
\begin{aligned}
& =-(p-1)^{p-1} \operatorname{div}\left(\frac{|\nabla u|^{p-2} \nabla u}{u^{p-1}}\right)-(p-1)^{p} \frac{|\nabla u|^{p}}{u^{p}} \\
& =-(p-1)^{p-1} \frac{|\nabla u|^{p-2}\langle\nabla f, \nabla u\rangle}{u^{p-1}} \\
& =|\nabla w|^{p-2}\langle\nabla f, \nabla w\rangle .
\end{aligned}
$$

Then we calculate directly

$$
\begin{aligned}
\operatorname{div}\left(h^{\frac{p}{2}-1} A(\nabla h)\right) & \\
=\left(\frac{p}{2}-1\right) & h^{\frac{p}{2}-2}|\nabla h|^{2}+h^{\frac{p}{2}-1} \Delta h+\left(\frac{p}{2}-2\right)(p-2) h^{\frac{p}{2}-3}\langle\nabla w, \nabla h\rangle^{2} \\
& +(p-2) h^{\frac{p}{2}-2}\langle\nabla w, \nabla h\rangle \Delta w+(p-2) h^{\frac{p}{2}-2}\langle\nabla\langle\nabla w, \nabla h\rangle, \nabla w\rangle .
\end{aligned}
$$

Using the standard Bochner type formula for $h=|\nabla w|^{2}$, namely

$$
\Delta h=2|\nabla \nabla w|^{2}+2 \operatorname{Ric}(\nabla w, \nabla w)+2\langle\nabla \Delta w, \nabla w\rangle,
$$

we have

(3-4) $\operatorname{div}\left(h^{\frac{p}{2}-1} A(\nabla h)\right)$

$$
\begin{array}{r}
=\left(\frac{p}{2}-1\right) h^{\frac{p}{2}-2}|\nabla h|^{2}+2 h^{\frac{p}{2}-1}\left(|\nabla \nabla w|^{2}+\operatorname{Ric}(\nabla w, \nabla w)+\langle\nabla \Delta w, \nabla w\rangle\right) \\
+\left(\frac{p}{2}-2\right)(p-2) h^{\frac{p}{2}-3}\langle\nabla w, \\
+\nabla h\rangle^{2}+(p-2) h^{\frac{p}{2}-2}\langle\nabla w, \nabla h\rangle \Delta w \\
+(p-2) h^{\frac{p}{2}-2}\langle\nabla\langle\nabla w, \nabla h\rangle, \nabla w\rangle .
\end{array}
$$

Rewrite (3-3) by using $h=|\nabla w|^{2}$ as

$$
h^{\frac{p}{2}-1} \Delta w+\left(\frac{p}{2}-1\right) h^{\frac{p}{2}-2}\langle\nabla h, \nabla w\rangle-h^{\frac{p}{2}}=h^{\frac{p}{2}-1}\langle\nabla f, \nabla w\rangle .
$$

Taking the gradient of both sides of (3-5) and then taking the product with $\nabla w$, we are led to

$$
\begin{aligned}
& \left(\frac{p}{2}-1\right)\left(\frac{p}{2}-2\right) h^{\frac{p}{2}-3}\langle\nabla w, \nabla h\rangle^{2}+\left(\frac{p}{2}-1\right) h^{\frac{p}{2}-2}\langle\nabla\langle\nabla w, \nabla h\rangle, \nabla w\rangle \\
& +h^{\frac{p}{2}-1}\langle\nabla \Delta w, \nabla w\rangle+\left(\frac{p}{2}-1\right) h^{\frac{p}{2}-2}\langle\nabla h, \nabla w\rangle \Delta w-\frac{p}{2} h^{\frac{p}{2}-1}\langle\nabla h, \nabla w\rangle \\
& =\left(\frac{p}{2}-1\right) h^{\frac{p}{2}-2}\langle\nabla f, \nabla w\rangle\langle\nabla h, \nabla w\rangle+h^{\frac{p}{2}-1}\langle\nabla\langle\nabla f, \nabla w\rangle, \nabla w\rangle .
\end{aligned}
$$

Adding (3-4) and twice (3-6) together and then simplifying, we have

$$
\begin{aligned}
& \operatorname{div}\left(h^{\frac{p}{2}-1} A(\nabla h)\right)-p h^{\frac{p}{2}-1}\langle\nabla h, \nabla w\rangle \\
& =\left(\frac{p}{2}-1\right) h^{\frac{p}{2}-2}|\nabla h|^{2}+2 h^{\frac{p}{2}-1}|\nabla \nabla w|^{2}+2 h^{\frac{p}{2}-1} \operatorname{Ric}(\nabla w, \nabla w) \\
& \quad+(p-2) h^{\frac{p}{2}-2}\langle\nabla f, \nabla w\rangle\langle\nabla h, \nabla w\rangle+2 h^{\frac{p}{2}-1}\langle\nabla\langle\nabla f, \nabla w\rangle, \nabla w\rangle .
\end{aligned}
$$


We also have

$$
\begin{aligned}
2 h^{\frac{p}{2}-1}\langle\nabla\langle\nabla f, \nabla w\rangle, & \nabla w\rangle \\
& =2 h^{\frac{p}{2}-1}(\nabla \nabla f)(\nabla w, \nabla w)+2 h^{\frac{p}{2}-1}(\nabla \nabla w)(\nabla f, \nabla w) \\
& =2 h^{\frac{p}{2}-1}(\nabla \nabla f)(\nabla w, \nabla w)+h^{\frac{p}{2}-1}\left\langle\nabla f, \nabla|\nabla w|^{2}\right\rangle \\
& =2 h^{\frac{p}{2}-1}(\nabla \nabla f)(\nabla w, \nabla w)+h^{\frac{p}{2}-1}\langle\nabla f, \nabla h\rangle .
\end{aligned}
$$

Moreover,

$$
\begin{aligned}
h^{\frac{p}{2}-1}\langle\nabla f, A(\nabla h)\rangle & =h^{\frac{p}{2}-1}\langle\nabla f, \nabla h\rangle+(p-2) h^{\frac{p}{2}-2}\langle\nabla f,(\nabla w \otimes \nabla w) \nabla h\rangle \\
& =h^{\frac{p}{2}-1}\langle\nabla f, \nabla h\rangle+(p-2) h^{\frac{p}{2}-2}\langle\nabla f, \nabla w\rangle\langle\nabla h, \nabla w\rangle .
\end{aligned}
$$

Now, (3-7) - (3-9) + (3-8) yields the desired result.

A maximum principle. When the triple $(M, g, f)$ is either shrinking or steady, Proposition 3.2 can be used to prove the following maximum principle.

Proposition 3.3. Let $u$ be a positive smooth solution to (3-1) in a bounded connected open subset $U$ in $M$ with smooth boundary $\partial U, p>1$. Suppose $(M, g, f)$ is a shrinking or steady gradient Ricci soliton. Then $|\nabla u| / u$ attains its maximum on $\partial U$.

Proof. Let $h=(p-1)^{2}|\nabla u|^{2} / u^{2}$. Assume $\max _{\bar{U}} h>\max _{\partial U} h$. Then there exists $x_{0} \in U$ such that $h\left(x_{0}\right)=\max _{\bar{U}} h>0$. Since $u \in C^{1, \alpha}$ and $u>0, h$ is continuous. Let

$$
V=\left\{x \in U: h(x)=h\left(x_{0}\right)\right\} .
$$

By the continuity of $h, V$ is a closed subset of $U$ and $V$ does not intersect $\partial U$. In fact, $h$ is positive and hence smooth in a neighborhood of $V$. There exists a point $x_{1} \in V$ such that for some $r_{0}$ the geodesic ball $B_{x_{1}}(r, g) \subset U$ is not contained in $V$ for any $0<r<r_{0}$, i.e., $x_{1}$ is a boundary point of $V$. By the continuity of $h$ again, there is a geodesic ball $B_{x_{1}}\left(r_{1}, g\right)$ in $U$ on which $h$ is positive. Observe that

$$
\begin{aligned}
\text { RHS of (3-2) } & =\frac{p-2}{2}|\nabla h|^{2} h^{\frac{p}{2}-2}+2 h^{\frac{p}{2}-1}|\nabla \nabla w|^{2}+2 h^{\frac{p}{2}-1}(\operatorname{Ric}+\nabla \nabla f)(\nabla w, \nabla w) \\
& \geq 2 h^{\frac{p}{2}-1}(\operatorname{Ric}+\nabla \nabla f)(\nabla w, \nabla w) \\
& = \begin{cases}2 h^{\frac{p}{2}-1}|\nabla w|^{2} \geq 0 & \text { if }(M, g, f) \text { is a shrinking soliton, } \\
0 & \text { if }(M, g, f) \text { is a steady soliton, }\end{cases}
\end{aligned}
$$

where for the first inequality, we argue as

$$
\begin{aligned}
4 h|\nabla \nabla w|^{2}+(p-2)|\nabla h|^{2} & \geq 4|\nabla w|^{2}|\nabla \nabla w|^{2}-\left.\left.|\nabla| \nabla w\right|^{2}\right|^{2} \\
& =4|\nabla w|^{2}\left(|\nabla \nabla w|^{2}-|\nabla| \nabla w||^{2}\right) \\
& \geq 0
\end{aligned}
$$


by Kato's inequality and $p \geq 1$. Then it follows that the linear differential operator $\mathcal{L}$ satisfies $\mathcal{L}(h) \geq 0$ on $U$. Next, since $A$ is positive definite and symmetric on $B_{x_{1}}\left(r_{1}, g\right)$, so is $h^{\frac{p}{2}-1} A$; therefore, $\mathcal{L}$ is uniformly elliptic on $B_{x_{1}}\left(r_{1}, g\right)$. By Hopf's strong maximum principle (see Theorem 3.5 in [Gilbarg and Trudinger 1998]), $h$ must be a constant on $B_{x_{1}}\left(r_{1}, g\right)$ since it attains its maximum at the interior point $x_{1}$. But this contradicts the maximality of $V$ as $B_{x_{1}}\left(r_{1}, g\right)$ contains points not in $V$.

Gradient estimates. Let us first recall the following gradient estimate:

Theorem 3.4 [Wang and Zhang 2011]. Let $\left(M^{n}, g\right)$ be a complete Riemannian manifold with Ric $\geq-(n-1) \kappa$ for some positive constant $\kappa$. Assume that $v$ is a positive $p$-harmonic function on the geodesic ball $B_{x_{0}}(R, g) \subset M$. Then

$$
\frac{|\nabla v|}{v} \leq C(p, n)\left(\frac{1}{R}+\sqrt{\kappa}\right)
$$

on $B_{x_{0}}\left(\frac{R}{2}, g\right)$ for some constant $C(p, n)$.

We now prove a gradient estimate for the $f$-weighted $p$-Laplacian equation.

Proposition 3.5. Let $\left(M^{n}, g, f\right)$ be a complete gradient Ricci soliton with

$$
\begin{aligned}
\left(\frac{2-p}{n-p}\right) \text { Ric } \geq-(n-1) \kappa e^{-2 f /(n-p)} g & \\
& -\frac{2 \varepsilon g}{n-p}-\frac{S g}{n-p}-\left(d f \otimes d f-|\nabla f|^{2} g\right) \frac{n-2}{(n-p)^{2}},
\end{aligned}
$$

where $S$ is the scalar curvature of $(M, g)$. Assume that $u$ is a positive solution of equation (3-1). Then there exists a constant $C(p, n)$ such that

$$
\frac{|\nabla u(x)|}{u(x)} \leq C(p, n)\left(\frac{1}{R}+\sqrt{\kappa}\right) e^{-f(x) /(n-p)}
$$

for $x \in B_{x_{0}}\left(\frac{R}{2}, e^{-2 f /(n-p)} g\right)$.

Proof. For a smooth function $f$, let $\nabla f$ be the gradient, $\Delta f$ the Laplacian, and $\nabla \nabla f$ the Hessian with respect to $g$. For the conformal change of metrics $\tilde{g}=e^{-2 f /(n-p)} g$, the Ricci tensors of $\tilde{g}$ and $g$ are related by

(3-11) $\widetilde{\text { Ric }}=\operatorname{Ric}-(n-2)\left(-\frac{\nabla \nabla f}{n-p}-\frac{d f \otimes d f}{(n-p)^{2}}\right)+\left(-\frac{\Delta f}{n-p}-\frac{n-2}{(n-p)^{2}}|\nabla f|^{2}\right) g$

(see [Anderson and Schoen 1985, p. 59]).

From the gradient Ricci soliton equation (2-1), the scalar curvature $S$ of $M$ satisfies the two equations

$$
\begin{aligned}
S+\Delta f-n \varepsilon & =0, \\
S+|\nabla f|^{2}+\varepsilon f & =0
\end{aligned}
$$

(see [Besse 1987]). 
Putting (2-1) and (3-12) into (3-11), we have

$$
\begin{aligned}
\widetilde{\operatorname{Ric}} & =\operatorname{Ric}+(n-2)\left(\frac{-\operatorname{Ric}-\varepsilon g}{n-p}+\frac{d f \otimes d f}{(n-p)^{2}}\right)+\left(\frac{S+n \varepsilon}{n-p}-\frac{n-2}{(n-p)^{2}}|\nabla f|^{2}\right) g \\
& =\frac{2-p}{n-p} \operatorname{Ric}+\frac{2 \varepsilon g}{n-p}+\frac{S g}{n-p}+\left(d f \otimes d f-|\nabla f|^{2} g\right) \frac{n-2}{(n-p)^{2}} .
\end{aligned}
$$

Therefore, the curvature assumption in Proposition 3.5 implies

$$
\widetilde{\operatorname{Ric}} \geq-(n-1) \kappa .
$$

By Proposition 3.1, we know that $u$ is also a positive solution to (1-1) for the metric $\tilde{g}$, hence by Theorem 3.4 we have

$$
\frac{|\nabla u|_{\tilde{g}}}{u} \leq C(p, n)\left(\frac{1}{R}+\sqrt{\kappa}\right)
$$

on $B_{x_{0}}\left(\frac{R}{2}, \tilde{g}\right)$. This is equivalent to

$$
\frac{|\nabla u(x)|}{u(x)} \leq C(p, n)\left(\frac{1}{R}+\sqrt{\kappa}\right) e^{-f(x) /(n-p)}
$$

for $x \in B_{x_{0}}\left(\frac{R}{2}, \tilde{g}\right)$.

A Liouville type theorem for the p-Laplace equation in dimension 2. For a steady gradient Ricci soliton, the condition (3-10) on the Ricci curvature in Proposition 3.5 cannot hold globally when $n \geq 3$ because it would imply, by taking the trace, that the scalar curvature is bounded below by a positive constant, which is impossible. However, the condition (3-10) is satisfied when $n=2$ for $p \geq 4$ or $1<p<2$ because

$$
\text { Ric }=\frac{1}{2} S g \geq \frac{1}{p-2} S g,
$$

since $S \geq 0$ for any steady gradient Ricci soliton [Chen 2009] and $\kappa=0$.

Note that Hamilton's cigar soliton is the unique 2-dimensional nonflat complete noncompact steady gradient Ricci soliton. The cigar soliton is $\mathbb{R}^{2}$ equipped with the complete metric

$$
g=\frac{d x^{2}+d y^{2}}{1+x^{2}+y^{2}}
$$

(see [Chow et al. 2006]) and the potential function

$$
f(x, y)=-\log \left(1+x^{2}+y^{2}\right) .
$$

The conformally altered metric is

$$
\tilde{g}=e^{2 \log \left(1+x^{2}+y^{2}\right) /(2-p)} g=\left(1+x^{2}+y^{2}\right)^{p /(2-p)}\left(d x^{2}+d y^{2}\right) .
$$


In particular, $\tilde{g}$ is complete if $1<p<2$ and incomplete if $p>2$. However, to use the gradient estimate in proving a Liouville type result, we will need $p \geq 4$. It is straightforward to compute the Gauss curvature of $\tilde{g}$ :

$$
\begin{aligned}
\widetilde{K} & =-\frac{1}{2}\left(1+r^{2}\right)^{p /(p-2)}\left(\partial_{r r}^{2}+\frac{1}{r} \partial_{r}\right) \log \left(1+r^{2}\right)^{-p /(p-2)} \\
& =\frac{2 p}{p-2}\left(1+r^{2}\right)^{(p /(p-2))-2} \\
& =\frac{2 p}{p-2}\left(1+r^{2}\right)^{-(p-4) /(p-2)}
\end{aligned}
$$

which is positive and tends to 0 as $r \rightarrow \infty$ if $p>4$. When $p=4$, the incomplete metric $\left(1+x^{2}+y^{2}\right)^{-2}\left(d x^{2}+d y^{2}\right)$ has constant curvature $\widetilde{K}=4$.

Theorem 3.6. Let $\left(\mathbb{R}^{2}, g, f\right)$ be Hamilton's cigar soliton. Then there does not exist any nonconstant positive $p$-harmonic function on $\left(\mathbb{R}^{2}, \tilde{g}\right)$ for $p \geq 4$.

Proof. Let $u$ be a positive solution to (3-1). For any point $x_{0} \in M$, the maximum principle (Proposition 3.3) asserts

$$
\frac{\left|\nabla u\left(x_{0}\right)\right|}{u\left(x_{0}\right)} \leq \max _{x \in \partial B_{0}(R, g)} \frac{|\nabla u(x)|}{u(x)}=\frac{\left|\nabla u\left(x_{R}\right)\right|}{u\left(x_{R}\right)}
$$

for some $x_{R} \in \partial B_{0}(R, g)$ where $x_{0} \in B_{0}(R, g)$ and $r\left(x_{0}, 0\right)<R$. From the discussion above, when $n=2$ and $p \geq 4$, the Ricci curvature condition (3-10) in Proposition 3.5 is satisfied. The diameter of $\left(\mathbb{R}^{2}, \tilde{g}\right)$ is

$$
2 R_{0}=2 \int_{0}^{\infty} \frac{d r}{\left(1+r^{2}\right)^{p /(2(p-2))}}<\infty .
$$

It is clear that $r\left(x_{R}, 0\right) \rightarrow \infty$ if and only if $\tilde{r}\left(x_{R}, 0\right) \rightarrow R_{0}$, where $\tilde{r}$ denotes the distance function for the metric $\tilde{g}$. Let

$$
r_{R}=\int_{R}^{\infty} \frac{d r}{\left(1+r^{2}\right)^{p /(2(p-2))}} .
$$

It follows from Proposition 3.5, applied on the ball $B_{x_{R}}\left(r_{R}, \tilde{g}\right)$, that

$$
\begin{aligned}
\frac{\left|\nabla u\left(x_{R}\right)\right|}{u\left(x_{R}\right)} & \leq C(n, p)\left(\frac{r_{x_{R}}}{2}\right)^{-1} e^{-2 \log \left(1+\left|x_{R}\right|^{2}\right) /(p-2)} \\
& =2 C(n, p)\left(\int_{R}^{\infty} \frac{d r}{\left(1+r^{2}\right)^{p /(2(p-2))}}\left(1+R^{2}\right)^{2 /(p-2)}\right)^{-1} \\
& \leq 2 C(n, p)\left(\left(1+R^{2}\right)^{2 /(p-2)} \int_{R}^{\infty} \frac{d r}{r^{p /(p-2)}}\right)^{-1} \\
& =2 C(n, p)\left(\frac{p-2}{2}\left(1+R^{2}\right)^{2 /(p-2)} R^{-2 /(p-2)}\right)^{-1}
\end{aligned}
$$


Since $p>2$, letting $R \rightarrow 0$ we conclude $\left|\nabla u\left(x_{0}\right)\right|=0$, hence $u$ is constant as $x_{0}$ is arbitrary.

\section{Acknowledgement}

Chen would like to thank Jiaping Wang for valuable discussions and his interest in this work.

\section{References}

[Ancona 1994] A. Ancona, "Convexity at infinity and Brownian motion on manifolds with unbounded negative curvature”, Rev. Mat. Iberoamericana 10:1 (1994), 189-220. MR 95a:58132 Zbl 0804.58056

[Anderson 1983] M. T. Anderson, "The Dirichlet problem at infinity for manifolds of negative curvature", J. Differential Geom. 18:4 (1983), 701-721. MR 85m:58178 Zbl 0541.53036

[Anderson and Schoen 1985] M. T. Anderson and R. Schoen, "Positive harmonic functions on complete manifolds of negative curvature", Ann. of Math. (2) 121:3 (1985), 429-461. MR 87a:58151 Zbl 0587.53045

[Besse 1987] A. L. Besse, Einstein manifolds, Ergebnisse der Mathematik und ihrer Grenzgebiete (3) 10, Springer, Berlin, 1987. MR 88f:53087 Zbl 0613.53001

[Cao and Zhou 2010] H.-D. Cao and D. Zhou, "On complete gradient shrinking Ricci solitons", J. Differential Geom. 85:2 (2010), 175-186. MR 2011k:53040 Zbl 1246.53051

[Chen 2009] B.-L. Chen, "Strong uniqueness of the Ricci flow", J. Differential Geom. 82:2 (2009), 363-382. MR 2010h:53095 Zbl 1177.53036

[Choi 1984] H. I. Choi, "Asymptotic Dirichlet problems for harmonic functions on Riemannian manifolds”, Trans. Amer. Math. Soc. 281:2 (1984), 691-716. MR 85b:53040 Zbl 0541.53035

[Chow et al. 2006] B. Chow, P. Lu, and L. Ni, Hamilton's Ricci flow, Graduate Studies in Mathematics 77, American Mathematical Society, Providence, RI, 2006. MR 2008a:53068 Zbl 1118.53001

[Dung and Dat 2015] N. T. Dung and N. D. Dat, "Local and global sharp gradient estimates for weighted $p$-harmonic functions", preprint, Vietnam Institute for Advanced Study in Mathematics, Ha Noi, 2015. arXiv 1505.07623

[Eberlein and O'Neill 1973] P. Eberlein and B. O'Neill, "Visibility manifolds", Pacific J. Math. 46 (1973), 45-109. MR 49 \#1421 Zbl 0264.53026

[Gilbarg and Trudinger 1998] D. Gilbarg and N. S. Trudinger, Elliptic partial differential equations of second order, revised 2nd ed., Grundlehren der Mathematischen Wissenschaften 224, Springer, Berlin, 1998. MR 2001k:35004 Zbl 1042.35002

[Hamilton 1995] R. S. Hamilton, "The formation of singularities in the Ricci flow", pp. 7-136 in Proceedings of the Conference on Geometry and Topology (Cambridge, MA, 1993), edited by C.-C. Hsiung and S.-T. Yau, Surveys in Differential Geometry 2, International Press, Cambridge, MA, 1995. MR 97e:53075 Zbl 0867.53030

[Holopainen 2002] I. Holopainen, "Asymptotic Dirichlet problem for the $p$-Laplacian on CartanHadamard manifolds", Proc. Amer. Math. Soc. 130:11 (2002), 3393-3400. MR 2003d:58055 Zbl 1016.58021

[Holopainen and Vähäkangas 2007] I. Holopainen and A. Vähäkangas, "Asymptotic Dirichlet problem on negatively curved spaces”, J. Anal. 15 (2007), 63-110. MR 2010j:58056 Zbl 1202.58017 
[Hsu 2003] E. P. Hsu, "Brownian motion and Dirichlet problems at infinity", Ann. Probab. 31:3 (2003), 1305-1319. MR 2004e:58063 Zbl 1054.58027

[Kotschwar and Ni 2009] B. Kotschwar and L. Ni, "Local gradient estimates of $p$-harmonic functions, 1/H-flow, and an entropy formula", Ann. Sci. École Norm. Sup. (4) 42:1 (2009), 1-36. MR 2010g:53121 Zbl 1182.53060

[Moser 2007] R. Moser, "The inverse mean curvature flow and $p$-harmonic functions", J. Eur. Math. Soc. 9:1 (2007), 77-83. MR 2007h:53102 Zbl 1116.53040

[Munteanu and Sesum 2013] O. Munteanu and N. Sesum, "On gradient Ricci solitons", J. Geom. Anal. 23:2 (2013), 539-561. MR 3023848 Zbl 1275.53061

[Munteanu and Wang 2012] O. Munteanu and J. Wang, "Analysis of weighted Laplacian and applications to Ricci solitons”, Comm. Anal. Geom. 20:1 (2012), 55-94. MR 2903101 Zbl 1245.53039

[Pansu 1989] P. Pansu, "Cohomologie $L^{p}$ des variétés à courbure négative, cas du degré 1 ", Rend. Sem. Mat. Univ. Politec. Torino Special Issue (1989), 95-120. MR 92e:58200 Zbl 0723.53023

[Sullivan 1983] D. Sullivan, “The Dirichlet problem at infinity for a negatively curved manifold", $J$. Differential Geom. 18:4 (1983), 723-732. MR 85m:58177 Zbl 0541.53037

[Sung and Wang 2014] C.-J. A. Sung and J. Wang, "Sharp gradient estimate and spectral rigidity for p-Laplacian”, Math. Res. Lett. 21:4 (2014), 885-904. MR 3275651 Zbl 1304.58018

[Tolksdorf 1984] P. Tolksdorf, "Regularity for a more general class of quasilinear elliptic equations", J. Differential Equations 51:1 (1984), 126-150. MR 85g:35047 Zbl 0488.35017

[Wang and Zhang 2011] X. Wang and L. Zhang, "Local gradient estimate for $p$-harmonic functions on Riemannian manifolds", Comm. Anal. Geom. 19:4 (2011), 759-771. MR 2880214 Zbl 1244.58007

[Wu 2013] P. Wu, "On the potential function of gradient steady Ricci solitons", J. Geom. Anal. 23:1 (2013), 221-228. MR 3010278 Zbl 1269.53051

[Yau 1975] S.-T. Yau, "Harmonic functions on complete Riemannian manifolds", Comm. Pure Appl. Math. 28 (1975), 201-228. MR 55 \#4042 Zbl 0291.31002

Received November 13, 2014. Revised September 3, 2015.

\section{JINGYI CHEN}

DEPARTMENT OF MATHEMATICS

UNIVERSITY OF BRITISH COLUMBIA

RoOM 121, 1984 MATHEMATICS ROAD

VANCOUVER BC V6T 1 Z2

CANADA

jychen@math.ubc.ca

YUE WANG

DEPARTMENT OF MATHEMATICS

CHINA JILIANG UNIVERSITY

HANGZHOU, ZHEJIANG 310018

CHINA

kellywong@cjlu.edu.cn 


\title{
PACIFIC JOURNAL OF MATHEMATICS
}

\author{
msp.org/pjm
}

Founded in 1951 by E. F. Beckenbach (1906-1982) and F. Wolf (1904-1989)

\section{EDITORS}

Don Blasius (Managing Editor)

Department of Mathematics

University of California

Los Angeles, CA 90095-1555

blasius@math.ucla.edu

\author{
Paul Balmer \\ Department of Mathematics \\ University of California \\ Los Angeles, CA 90095-1555 \\ balmer@math.ucla.edu \\ Robert Finn \\ Department of Mathematics \\ Stanford University \\ Stanford, CA 94305-2125 \\ finn@math.stanford.edu \\ Sorin Popa \\ Department of Mathematics \\ University of California \\ Los Angeles, CA 90095-1555 \\ popa@math.ucla.edu
}

\author{
Vyjayanthi Chari \\ Department of Mathematics \\ University of California \\ Riverside, CA 92521-0135 \\ chari@math.ucr.edu \\ Kefeng Liu \\ Department of Mathematics \\ University of California \\ Los Angeles, CA 90095-1555 \\ liu@math.ucla.edu \\ Jie Qing \\ Department of Mathematics \\ University of California \\ Santa Cruz, CA 95064 \\ qing@ cats.ucsc.edu
}

\section{PRODUCTION}

Silvio Levy, Scientific Editor, production@msp.org

\section{SUPPORTING INSTITUTIONS}

ACADEMIA SINICA, TAIPEI

CALIFORNIA INST. OF TECHNOLOGY

INST. DE MATEMÁTICA PURA E APLICADA

KEIO UNIVERSITY

MATH. SCIENCES RESEARCH INSTITUTE

NEW MEXICO STATE UNIV.

OREGON STATE UNIV.

\author{
STANFORD UNIVERSITY \\ UNIV. OF BRITISH COLUMBIA \\ UNIV. OF CALIFORNIA, BERKELEY \\ UNIV. OF CALIFORNIA, DAVIS \\ UNIV. OF CALIFORNIA, LOS ANGELES \\ UNIV. OF CALIFORNIA, RIVERSIDE \\ UNIV. OF CALIFORNIA, SAN DIEGO \\ UNIV. OF CALIF., SANTA BARBARA
}

\author{
Daryl Cooper \\ Department of Mathematics \\ University of California \\ Santa Barbara, CA 93106-3080 \\ cooper@math.ucsb.edu \\ Jiang-Hua Lu \\ Department of Mathematics \\ The University of Hong Kong \\ Pokfulam Rd., Hong Kong \\ jhlu@maths.hku.hk \\ Paul Yang \\ Department of Mathematics \\ Princeton University \\ Princeton NJ 08544-1000 \\ yang@math.princeton.edu
}

These supporting institutions contribute to the cost of publication of this Journal, but they are not owners or publishers and have no responsibility for its contents or policies.

See inside back cover or msp.org/pjm for submission instructions.

The subscription price for 2016 is US $\$ 440 /$ year for the electronic version, and $\$ 600 /$ year for print and electronic.

Subscriptions, requests for back issues and changes of subscriber address should be sent to Pacific Journal of Mathematics, P.O. Box 4163, Berkeley, CA 94704-0163, U.S.A. The Pacific Journal of Mathematics is indexed by Mathematical Reviews, Zentralblatt MATH, PASCAL CNRS Index, Referativnyi Zhurnal, Current Mathematical Publications and Web of Knowledge (Science Citation Index).

The Pacific Journal of Mathematics (ISSN 0030-8730) at the University of California, c/o Department of Mathematics, 798 Evans Hall \#3840, Berkeley, CA 94720-3840, is published twelve times a year. Periodical rate postage paid at Berkeley, CA 94704, and additional mailing offices. POSTMASTER: send address changes to Pacific Journal of Mathematics, P.O. Box 4163, Berkeley, CA 94704-0163.

PJM peer review and production are managed by EditFLOW ${ }^{\circledR}$ from Mathematical Sciences Publishers.

\section{PUBLISHED BY}

\section{mathematical sciences publishers \\ nonprofit scientific publishing}

http://msp.org/

(C) 2016 Mathematical Sciences Publishers 


\section{PACIFIC JOURNAL OF MATHEMATICS}

Volume $282 \quad$ No. $2 \quad$ June 2016

Exhausting curve complexes by finite rigid sets

JAVIER ARAMAYONA and CHRISTOPHER J. LEININGER

A variational characterization of flat spaces in dimension three

Giovanni Catino, Paolo Mastrolia and Dario D. Monticelli

Estimates of the gaps between consecutive eigenvalues of Laplacian

293

DAgUang Chen, TAO Zheng and HongCANG YANG

Liouville type theorems for the $p$-harmonic functions on certain manifolds

JINGYI CHEN and YUE WANG

Cartan-Fubini type rigidity of double covering morphisms of quadratic manifolds

\section{HOSUNG KIM}

On the uniform squeezing property of bounded convex domains in $\mathbb{C}^{n}$

KANG-TAE KIM and LIYOU ZHANG

Lefschetz pencils and finitely presented groups

RYOMA KOBAYASHI and NAOYUKI MONDEN

Knot homotopy in subspaces of the 3-sphere

YUYA KODA and MAKOTO OzAWA

On the relationship of continuity and boundary regularity in prescribed mean curvature Dirichlet problems

KIRK E. LANCASTER and JARON MELIN

Bridge spheres for the unknot are topologically minimal

JUNG HOON LEE

On the geometric construction of cohomology classes for cocompact discrete subgroups of $\mathrm{SL}_{n}(\mathbb{R})$ and $\mathrm{SL}_{n}(\mathbb{C})$

SUSANNE SCHIMPF

On Blaschke's conjecture

Xiaole Su, Hongwei Sun and Yusheng Wang

The role of the Jacobi identity in solving the Maurer-Cartan structure equation 